\title{
Correlação da topografia tumoral e edema peritumoral com a resposta terapêutica à administração intranasal do álcool perílico nos gliomas malignos recidivos
}

\author{
Julio Thomé Souza Silva', Raphael Moreira Texeira², Thereza Quirico-Santos ${ }^{3}$,
} Clovis Orlando da Fonseca ${ }^{4}$

Faculdade de Medicina da Universidade Federal Fluminense, Rio de Janeiro, RJ, Brasil

Instituto de Biologia da Universidade Federal Fluminense, Rio de Janeiro, RJ, Brasil

\section{RESUMO}

Objetivo: Avaliar a correlação da topografia tumoral e edema peritumoral com a resposta terapêutica à administração intranasal do álcool perílico (AP) em uma coorte de pacientes com gliomas malignos recidivos. Métodos: Os autores revisaram retrospectivamente 67 pacientes com gliomas malignos recidivos que receberam administração intranasal de $440 \mathrm{mg}$ de AP diariamente. Parâmetros avaliados incluíram aspectos clínicos e os de neuroimagem. Avaliação clínica incluiu dados demográficos, sintomas iniciais e sobrevida global. Dados de imagem incluíram topografia tumoral, volume tumoral, presença de desvio da linha média e extensão de edema peritumoral. Análise bioestatística foi realizada usando testes log rank. Sobrevida global foi medida e analisada pelo método de Kaplan Meier, incluindo intervalos de confiança de $95 \%$. Resultados: Um total de 67 pacientes foi investigado, 52 (77,6\%) com glioblastoma (GBM), 10 (14,9\%) com astrocitoma anaplásico $(A A)$ e $5(7,4 \%)$ com oligodendrioglioma anaplásico (OA). Todos os cinco pacientes com OA apresentaram tumor de localização lobar. Nos $A A$, oito casos estavam localizados em região talâmica e dois em região lobar, enquanto que, nos GBM, 11 casos de localização talâmica e 41 lobares. A relação volume tumoral e edema peritumoral foi observada. Pacientes com regressão tumoral e edema peritumoral apresentaram resposta positiva enquanto que aqueles com regressão tumoral sem regressão do edema peritumoral não apresentaram boa evolução clínica. Pacientes com gliomas profundos sobreviveram significativamente mais tempo do que aqueles com gliomas lobares (log rank test, $p=0,0003$ ). Presença de desvio da linha média $(>1 \mathrm{~cm})$ foi estatisticamente significante como fator de risco para a sobrevida mais curta (log rank test, $p=0,0062)$. Conclusões: Este estudo sugere que: (1) pacientes com gliomas recidivos de localização profunda, estatisticamente, sobreviveram mais do que aqueles com tumores de localização lobar; (2) edema peritumoral é determinante na sintomatologia, provavelmente implicado na morbidade e pode estar relacionado com a característica invasiva dos gliomas malignos. Esses achados corroboram a teoria de que a interação entre as células dos gliomas e diferentes microambientes cerebral pode influenciar a fisiopatologia tumoral, resultando no desequilibrio da homeostase tecidual e contribuindo para a piora do prognóstico.

\section{PALAVRA-CHAVE}

Gliomas. Administração intranasal, Álcool perílico.

\section{ABSTRACT}

Correlation of tumor topography and peritumoral edema with therapeutic response to a new approach for recurrent malignant gliomas, intranasal administration of perillyl alcohol

Objective: To evaluate the correlation of tumor topography and peritumoral brain edema with the therapeutic response of intranasal administration of perillyl alcohol $(\mathrm{POH})$ in a cohort of patients with recurrent malignant gliomas. Methods: We retrospectively reviewed 67 consecutive patients with recurrent malignant gliomas who received administration intranasal of $\mathrm{POH} 440 \mathrm{mg}$ daily. The parameters evaluated were clinical features and the neuroimaging findings. Clinical data included demographics, initial symptoms, and overall survival. Imaging analysis included tumor topography, tumor size, presence of midline shift and extent of peritumoral edema. Biostatistics was carried out using log rank tests. Overall survival was measure and analyzed by Kaplan Meier method including 95\% confidence intervals. A total of 67 patients were investigated, 52 (77.6\%) with glioblastoma (GBM), $10(14.9 \%)$ with anaplastic astrocytoma (AA) and $5(7.4 \%)$ with anaplastic oligodendroglioma (AO). All five $A O$ had lobar localization, $A A$ were lobar in 8 cases and deep

1 Mestrando do curso de pós-graduação de Ciências Médicas da Universidade Federal Fluminense, Niterói, RJ.

2 Graduando da Faculdade de Medicina da Universidade Federal Fluminense, Niterói, RJ.

3 Professora titular do Departamento de Biologia Celular e Molecular do Instituto de Biologia da Universidade Federal Fluminense, Niterói, RJ.

4 Professor associado do Serviço de Neurocirurgia da Faculdade de Medicina da Universidade Federal Fluminense, Niterói, RJ. 
in 2 cases, whereas GBM were lobar in 41 cases and deep in 11 cases. Results: A relationship between the tumor size and the volume of peritumoral brain edema (PTBE) was observed. Patients with regression of the tumor and PTBE had positive response whereas those with regression of the tumor without PTBE regression had poor prognosis. Patients with deep tumors survived significantly longer than those with lobar gliomas (log rank test, $p=0.0003)$. Presence of midline shift $(>1 \mathrm{~cm}$ ) was a statistically significant risk factor for shorter survival (log rank test, $p=0.0062)$. Conclusions: This study suggests that: 1) patients with deep localization in recurrent gliomas may survive significantly longer than those with tumors in a lobar localization; 2) presence of PTBE contributes strongly to symptoms, likely implicated in the morbidity and may be related to the invading potential of malignant gliomas. These findings may corroborate the theory of that interaction between glioma cells and different brain microenvironment can influence glioma pathobiology, resulting in the disruption of tissue homeostasis contributing to its poor prognosis.

\section{KEY WORDS}

Gliomas. Intranasal administration. Perillyl alcohol.

\section{Introdução}

Tumores astrocíticos dividem-se em duas classes distintas de acordo com a interação com o microambiente cerebral: localizados e difusos. ${ }^{1}$ Os astrocitomas localizados têm um padrão de crescimento circunscrito e limitado potencial invasivo, diferentemente dos astrocitomas difusos, que são caracterizados por uma elevada capacidade infiltrativa. ${ }^{3}$ Gliomas malignos, o subtipo mais comum de tumores primários cerebrais, são agressivos, altamente invasivos e contêm células neoplásicas e tecido estromal, o que contribui para sua heterogeneidade histológica. ${ }^{17} \mathrm{O}$ crescimento da massa tumoral promove forte manifestação gliótica dos astrócitos normais que circundam o tumor - gliose reativa -, extinguindo um limite distinto do tumor a partir de tecido normal adjacente. Os exames de neuroimagem podem visualizar áreas com alto percentual de células tumorais e intensa neuroangiogênese, mas não áreas com baixa densidade de invasão de células tumorais. ${ }^{18}$ Portanto um dos impedimentos para o tratamento dos gliomas malignos tem sido o grau com que se expandem, infiltrando tecido circundante e ambiente peritumoral, tornando ineficaz a ressecção, a radioterapia, a quimioterapia ou a terapia genética. ${ }^{16}$

Autores usando modelo biomatemático de proliferação e migração dos gliomas hipotetisaram que o crescimento desses tumores na substância cinzenta profunda cerebral teria um intervalo de tempo maior comparado ao de lesões situadas na substância branca lobar, onde a invasão e a migração seriam mais rápidas..$^{10,23}$ Células gliais transformadas que se caracterizam por processos de proliferação e neoangiogênese preferencialmente invadem estruturas anatômicas, como axônios mielinizados, membrana basal vascular e subepêndima, interagindo especificamente com seu microambiente. ${ }^{14}$ Essas interações também podem desempenhar um papel na sobrevivência do paciente: pacientes com glioblas- toma multiforme localizado na substância cinzenta profunda sobrevivem estatisticamente por mais tempo do que pacientes com glioma lobar, independentemente do volume do tumor. ${ }^{21}$ Tem-se teorizado que essa diferença de sobrevida reflete a capacidade diferencial de invasão de células de gliomas que estão localizadas em diferentes microambientes. Portanto, em razão da eloquência biológica da massa cinzenta profunda do cérebro (tálamo e núcleos da base), pode-se esperar que gliomas profundos apresentem manifestações clínicas mais precoces. ${ }^{21}$

Edema peritumoral é determinante na sintomatologia causada pelos gliomas de alto grau caracterizando-se por extensa infiltração de células tumorais. ${ }^{24}$ Causa adicional efeito de massa, resultando em aumento da pressão intracraniana, acarretando déficits neurológicos por causa da desregulação da homeostase tecidual e da redução do fluxo sanguíneo cerebral. Por haver correlação positiva entre a presença de edema peritumoral com menor sobrevida e alta recidiva em pacientes portadores de gliomas malignos, é razoável considerar a presença de edema como um fator adicional a ser levado em conta ao traçar estratégias para o tratamento desses tumores. ${ }^{12}$ Embora a introdução de corticosteroides tenha simplificado o tratamento de pacientes com tumores iniciais, seu uso de forma prolongada é um problema crucial no contexto de gliomas malignos recidivos associados a edema, em razão de efeitos colaterais marcantes. Portanto, estudos que visam ao entendimento do processo evolutivo do edema peritumoral com ajuda da metodologia molecular tornam-se necessários; por exemplo, pesquisa mostra que a inibição do receptor do fator de crescimento vascular endotelial (VEGF) pode ser uma via para estabelecer estratégia terapêutica para a resolução do edema peritumoral. ${ }^{25}$

Em nossos estudos clínicos ${ }^{4-7}$ estamos observando que a localização dos gliomas e a presença de edema peritumoral são fatores determinantes de sobrevida e recidiva tumoral. 
O presente estudo objetiva investigar a correlação da resposta terapêutica do álcool perílico (AP) intranasal com a topografia tumoral e o edema peritumoral em uma série clínica de pacientes adultos com gliomas recidivos. Em virtude da compreensão das etapas de proliferação e migração serem importantes para buscar diretrizes específicas para procedimentos terapêuticos, procuramos discutir a influência do microssistema, ou seja, do estroma tumoral para a progressão e a invasão dos gliomas malignos.

\section{Pacientes e métodos}

A coorte foi composta de 67 pacientes com gliomas malignos recidivos, após terapia convencional, sendo $52(77,6 \%)$ com glioblastoma multiforme (GBM), 10 $(14,9 \%)$ com astrocitoma anaplásico (AA) e $5(7,4 \%)$ com oligodendroglioma anaplásico $(\mathrm{OA})$. O projeto foi aprovado pelo Conselho Nacional de Ética em Pesquisa (Conep), com registro número Conep 9681 n $^{\circ}$ 25000.009267/2004-25, aprovado em julho de 2004.

\section{Critérios para inclusão}

Foram incluídos pacientes com glioma maligno recidivo com idade entre 20 e 70 anos e escore Karnofisky $\geq 70$, independentemente de gênero, etnia e tempo de recidiva do tumor. Como critérios de elegibilidade foram considerados: paciente ter sido submetido a tratamento prévio convencional específico para este tipo de tumor (ablação cirúrgica do tumor, radioterapia e/ou quimioterapia); os valores basais dos exames de rotina, bioquímica do sangue e hematológicos; a avaliação cardiológica (ritmo cardíaco sinusal); avaliação de imagem pulmonar (ausência de lesão parenquimatosa). Os critérios de inclusão foram seguidos de modo rigoroso.

\section{Critérios de inelegibilidade}

Não foram incluídos: pacientes com doença maligna sistêmica; mulheres grávidas ou amamentando; tratamento prévio com qualquer droga experimental nos últimos 90 dias; tratamento concomitante com droga experimental; metástase a distância; alteração nas provas de função hematológica, renal, cardiovascular ou hepática; infecção em atividade; presença de doença concomitante sem controle clínico adequado, que possa interferir na participação do estudo; infarto do miocárdio há menos de seis meses.

\section{Métodos}

Os parâmetros avaliados incluíram as características clínicas e os achados em neuroimagens. Os sintomas iniciais de cada paciente foram relacionados com a localização do tumor no sistema nervoso central e com apresentação clínica. As variáveis clínicas foram: histologia (OA, AA ou GBM), tratamento prévio (biópsia ou excisão cirúrgica, radioterapia ou quimioterapia) e sobrevida global após a recidiva. Nas imagens de tomografia computadorizada (TC) ou ressonância magnética (RM), o local (lobar ou profundo) e o tamanho $\left(\mathrm{em} \mathrm{cm}^{2}\right)$ foram registrados. Além disso, a extensão do edema peritumoral e os efeitos de massa-desvio da linha média (todos em $\mathrm{cm}$ ) foram avaliados no início e no decorrer do tratamento com a administração intranasal do AP. Tumores lobares foram definidos como qualquer lesão tumoral, que não envolvem os gânglios basais. Todos os tumores limitados aos gânglios da base ou envolvendo-os foram classificados como lesões de substância cinzenta profunda. O tamanho do tumor foi mensurado em reforço de contraste-axial usando a escala dos maiores diâmetros perpendiculares da lesão. O tamanho do edema peritumoral foi medido nos mesmos cortes axiais utilizados para medições do tamanho do tumor, subtraindo o tumor do diâmetro do edema. A comparação foi mantida constante com o mesmo tipo de imagem axial (TC ou ressonância magnética). Análise bioestatística foi realizada utilizando curvas de Kaplan Meier, log rank e modelos de regressão Cox multivariada. A associação das variáveis categóricas com a sobrevida foi avaliada utilizando curvas de Kaplan Meier e teste log rank, e o significado das variáveis contínuas foi avaliado utilizando modelos de regressão Cox univariada. As variáveis que foram associadas com a sobrevida em análise univariada $(\mathrm{p}=0,3)$ foram consideradas potenciais para inclusão no modelo multivariado.

\section{Resultados}

Os pacientes incluídos no estudo foram assim distribuídos: total de 67 pacientes, 40 homens e 37 mulheres, com idade variando de 28 a 69 anos; 52 pacientes com GBM, 10 com AA e 5 pacientes com OA. Todos os pacientes foram tratados previamente com cirurgia, radioterapia e quimioterapia. A distribuição das queixas iniciais (múltiplos sintomas possíveis) nos tumores iniciais está apresentada no gráfico 1 . As demais características estudadas estão apresentadas na tabela 1 . 


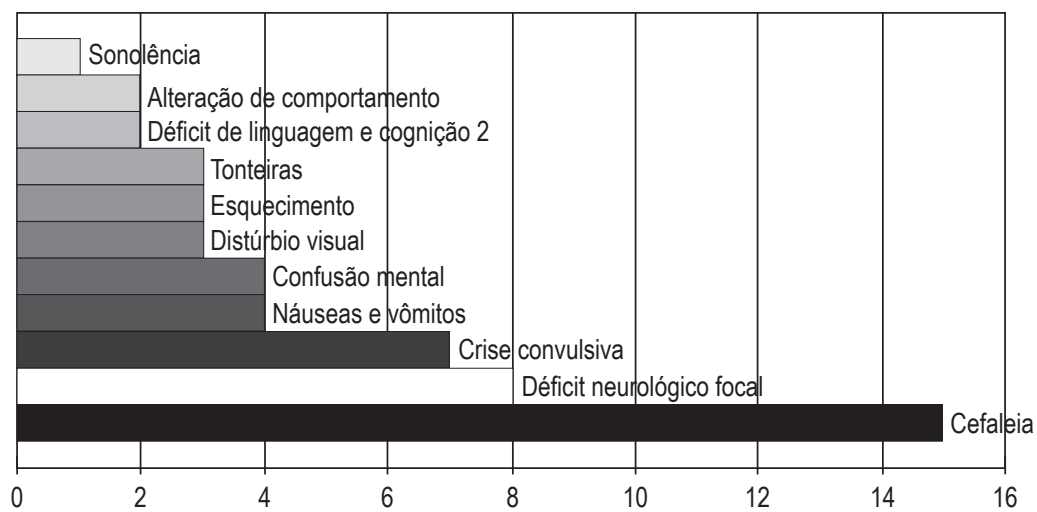

Gráfico 1 - Sintomas apresentados em pacientes portadores de gliomas malignos em porcentagem.

\begin{tabular}{|c|c|c|c|}
\hline \multicolumn{4}{|c|}{$\begin{array}{c}\text { Tabela } 1 \\
\text { Características dos pacientes }(n=67)\end{array}$} \\
\hline & GBM & AA & OA \\
\hline Total e pacientes & 52 & 10 & 5 \\
\hline $\begin{array}{l}\text { Idade } \\
\text { Média } \\
\text { Limite }\end{array}$ & $\begin{array}{c}50 \\
38-62\end{array}$ & $\begin{array}{c}52,5 \\
41-64\end{array}$ & $\begin{array}{c}48.9 \\
28-72\end{array}$ \\
\hline $\begin{array}{l}\text { Gênero } \\
\text { Homens } \\
\text { Mulheres }\end{array}$ & $\begin{array}{l}31 \\
21\end{array}$ & $\begin{array}{l}6 \\
4\end{array}$ & $\begin{array}{l}3 \\
2\end{array}$ \\
\hline $\begin{array}{l}\text { Sítio primário do tumor } \\
\text { Lobo frontal } \\
\text { Lobo temporal } \\
\text { Lobo perietal } \\
\text { Lobo occipital } \\
\text { Cerebelo }\end{array}$ & $\begin{array}{c}21 \\
16 \\
11 \\
4 \\
0\end{array}$ & $\begin{array}{l}3 \\
3 \\
3 \\
1 \\
0\end{array}$ & $\begin{array}{l}3 \\
1 \\
1 \\
0 \\
0\end{array}$ \\
\hline $\begin{array}{l}\text { Sintomas iniciais predominantes } \\
\text { Cefaleia } \\
\text { Crise convulsiva } \\
\text { Déficit neurológico focal } \\
\text { Déficit temporário de memória } \\
\text { Confusão mental } \\
\text { Alteração de comportamento }\end{array}$ & $\begin{array}{c}53,57 \% \\
25 \% \\
48,57 \% \\
10,71 \% \\
14,28 \% \\
7,14 \%\end{array}$ & $\begin{array}{c}55,27 \% \\
22 \% \\
49,43 \% \\
13,11 \% \\
9,15 \% \\
8,64 \%\end{array}$ & $\begin{array}{c}49 \% \\
65 \% \\
8 \% \\
12,46 \% \\
36 \% \\
7 \%\end{array}$ \\
\hline $\begin{array}{l}\text { Tratamento de tumor inicial } \\
\text { Ablação cirúrgica } \\
\text { Biópsia } \\
\text { Radioterapia } \\
\text { Quimioterapia }\end{array}$ & $\begin{array}{c}78 \% \\
22 \% \\
100 \% \\
100 \%\end{array}$ & $\begin{array}{c}77 \% \\
23 \% \\
100 \% \\
100 \%\end{array}$ & $\begin{array}{l}100 \% \\
0 \% \\
100 \% \\
100 \%\end{array}$ \\
\hline $\begin{array}{l}\text { Sobrevida livre de progressão } \\
\quad\left(6^{\circ} \text { mês }\right)\end{array}$ & $48,2 \%$ & $60 \%$ & $66,6 \%$ \\
\hline $\begin{array}{l}\text { Edema no tumor inicial (RM) } \\
\text { Ausente } \\
<5 \mathrm{~cm} \\
>5 \mathrm{~cm}\end{array}$ & $\begin{array}{c}5(9,6 \%) \\
24(46,2 \%) \\
28(53,8 \%)\end{array}$ & $\begin{array}{l}1(10 \%) \\
7(70 \%) \\
2(20 \%)\end{array}$ & $\begin{array}{c}3(60 \%) \\
2(40 \%) \\
0\end{array}$ \\
\hline $\begin{array}{l}\text { Edema no tumor recidivo (RM) } \\
\quad<5 \mathrm{~cm} \\
\quad>5 \mathrm{~cm}\end{array}$ & $\begin{array}{l}19(36,5 \%) \\
33(63,4 \%)\end{array}$ & $\begin{array}{l}6(60 \%) \\
4(40 \%)\end{array}$ & $\begin{array}{c}5(100 \%) \\
0\end{array}$ \\
\hline $\begin{array}{l}\text { Desvio de linha média no } \\
\text { tumor inicial (RM) } \\
\text { Ausente } \\
<1 \mathrm{~cm} \\
>1 \mathrm{~cm}\end{array}$ & $\begin{array}{l}8(15,3 \%) \\
19(36,5 \%) \\
25(48,7 \%)\end{array}$ & $\begin{array}{l}2(20 \%) \\
6(60 \%) \\
2(20 \%)\end{array}$ & $\begin{array}{c}3(60 \%) \\
2(40 \%) \\
0\end{array}$ \\
\hline $\begin{array}{l}\text { Desvio de linha média no } \\
\text { tumor recidivo (RM) } \\
\text { Ausente } \\
<1 \mathrm{~cm} \\
>1 \mathrm{~cm}\end{array}$ & $\begin{array}{c}0 \\
18(35,5 \%) \\
34(64,5 \%) \\
\end{array}$ & $\begin{array}{c}0 \\
4(40 \%) \\
6(60 \%)\end{array}$ & $\begin{array}{c}0 \\
3(60 \%) \\
2(40 \%)\end{array}$ \\
\hline
\end{tabular}

A análise de sobrevida nos três grupos (definidos como GBM, AA e OA) diferiu significativamente (Figura 1).

Sobrevida em pacientes com AA e GBM localizados nos gânglios basais foi significativamente maior que aqueles com lesões lobares (teste log rank: $\mathrm{p}=0,0003$ ) (Figura 2). Pacientes com edema cerebral extenso $(>5 \mathrm{~cm})$ apresentaram sobrevida menor que aqueles com edema moderado (Figura 3). Presença de desvio de linha média $(>1 \mathrm{~cm})$ foi fator de risco estatisticamente significante para sobrevida menor (Figura 4). Não foi observada diferença significativa na sobrevida analisada pela extensão do procedimento cirúrgico (biópsia versus ressecção parcial ou total).
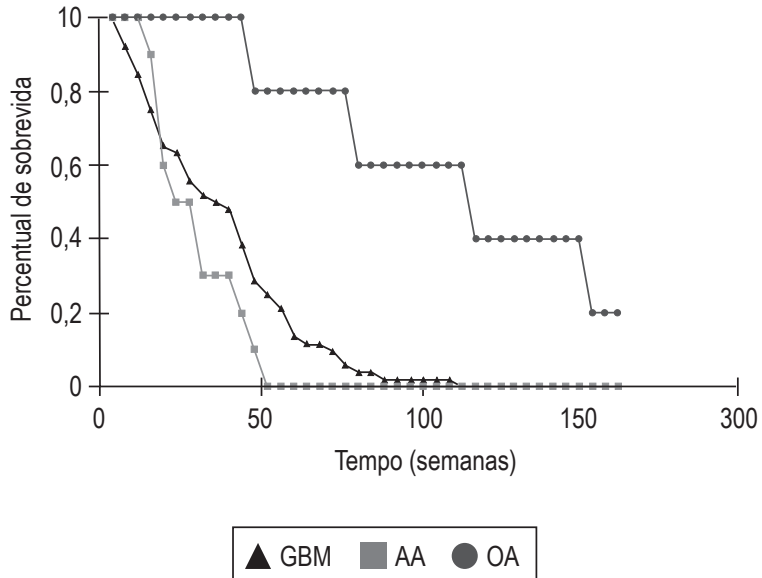

Figura 1 - Análise de sobrevida por meio do estimador de Kaplan-Meier em pacientes portadores de gliomas malignos recidivos tratados com $A P$ intranasal. 


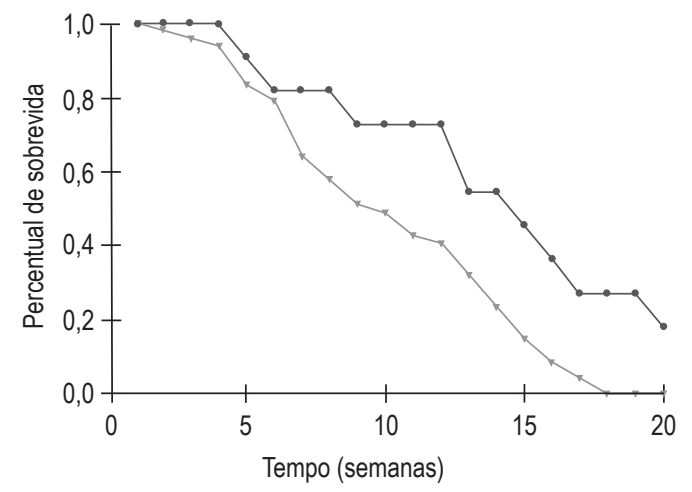

PROFUNDO $\nabla$ LOBAR

Figura 2 - Estimador de Kaplan-Meier mostrando diferença de sobrevida de acordo com a localização (lobar versus talâmico) em pacientes com gliomas malignos recidivos tratados com AP intranasal.

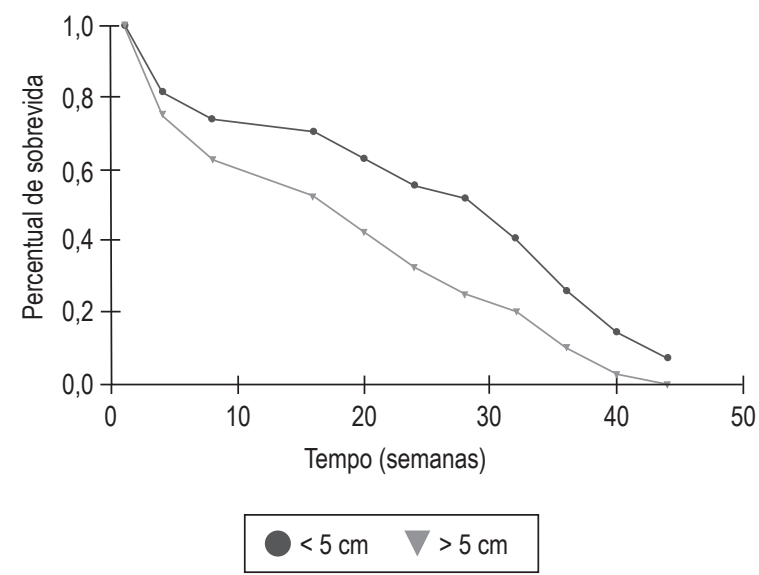

Figura 3 - Estimador de Kaplan-Meier mostrando diferença de sobrevida de acordo com a extensão do edema peritumoral em pacientes com gliomas malignos recidivos tratados com AP intranasal.



Figura 4-Estimador de Kaplan-Meier mostrando diferença de sobrevida de acordo com o desvio da linha média em pacientes com gliomas malignos recidivos tratados com AP intranasal.

\section{Discussão}

A observação mais importante desse estudo é que pacientes com tumores localizados em região talâmica e nos gânglios basais, independentemente do grau histológico, apresentam sobrevida maior que aqueles com tumores em localização lobar. Além disso, a análise da presença de edema peritumoral associada à presença de desvio da linha média foi estatisticamente significante.

Embora os gliomas malignos não produzam metástases, as células tumorais migram ativamente através do parênquima cerebral e, assim, fazem surgir novos focos tumorais tornando-os imperceptíveis para ressecção cirúrgica eficaz. ${ }^{22}$ Estudos mostram que o crescimento da massa tumoral promove forte manifestação gliótica dos astrócitos normais que circundam o tumor - gliose reativa. ${ }^{2}$ Acredita-se que a migração das células tumorais aumente com o tempo, embora a densidade de células infiltrantes possa diminuir com a distância a partir da massa tumoral inicial. ${ }^{14}$ De fato, quando os gliomas recidivam após o tratamento, geralmente o fazem a cerca de $2 \mathrm{~cm}$ do local original. ${ }^{15}$ Atribui-se o desenvolvimento tumoral à influência do microambiente - estroma - que produz fatores de crescimento, citocinas e matriz extracelular e também participa no recrutamento de células endoteliais necessárias para a neovascularização do tumor. ${ }^{2}$ Hoelzinger e col. ${ }^{11}$ enfatizam que o processo bioquímico da migração dos gliomas inclui sinalizações autócrinas transmitidas por fatores de crescimento modulados por meio de receptores no tumor. Esses fatores secretados podem se difundir por intermédio do estroma peritumoral influindo células do parênquima que circundam a massa tumoral. Reciprocamente, células do parênquima cerebral normal que secretam ligantes podem estimular receptores em células de gliomas facilitando a migração ou criar um microambiente permissivo para progressão maligna. Essas interações possivelmente refletem a diferença do processo migratório das células de gliomas em distintos microambientes, influenciando a sobrevida dos pacientes: aqueles portadores de GBM localizado na região talâmica e gânglios da base sobrevivem estatisticamente mais tempo do que pacientes com glioma lobar. ${ }^{21}$

Swanson e col. ${ }^{23}$ estudando modelos matemáticos para quantificar crescimento e invasividade de gliomas concluíram que: (1) a velocidade de expansão é linear com o tempo, variando de cerca de $4 \mathrm{~mm} /$ ano para o glioma de baixo grau para cerca de $3 \mathrm{~mm} / \mathrm{mês}$ nos casos de glioma de alto grau; (2) as células de glioma migram mais lentamente em substância cinzenta profunda em comparação com substância branca de localização lobar. Esses autores também sugerem que invasividade dos gliomas requer a conjunção de conceitos que incluem 
motilidade celular e crescimento proliferativo. Nossas observações estão consoantes com esses resultados: pacientes com gliomas malignos inicialmente localizados nos gânglios basais sobrevivem significativamente mais do que aqueles com gliomas em região lobar. Também observamos que em tumores lobares a presença de edema peritumoral é maior que em tumores de localização profunda, mas são necessários mais estudos de imagem para confirmação.

Publicações da última década acerca da sobrevida em pacientes com gliomas malignos são retrospectivas e os fatores associados a prognóstico favorável são: menor faixa etária, ausência de déficit neurológico, grau histológico e localização em áreas não eloquentes. ${ }^{16}$ Estudo observacional ${ }^{13}$ fornece dados que indicam ressecção cirúrgica como fator importante na sobrevida de pacientes com gliomas malignos. Enquanto gliomas lobares podem ser tratados com excisão cirúrgica, autores ${ }^{19}$ recomendam biópsia estereotáxica para gliomas talâmicos porque os resultados não demonstraram melhoria significativa na sobrevida após ressecção extensa. Em nossa série, o grande número de pacientes com gliomas talâmicos iniciais não foi tratado com cirurgia citorredutora enquanto pacientes com localização lobar foram submetidos à excisão cirúrgica.

Em um estudo retrospectivo, ${ }^{20}$ realizado em 145 pacientes operados (102 com GBM, e 43 com AA), os autores concluíram que o resultado após tratamento cirúrgico depende de idade dos pacientes e condições clínicas pré-operatórias, assim como a localização, a extensão da lesão e a extensão da ressecção do tumor. Em nossa série $78 \%$ dos pacientes com GBM, 77\% dos pacientes com AA e $100 \%$ dos pacientes com OA foram submetidos a tratamento cirúrgico. Ressecção radical foi realizada em $46,3 \%$ e parcial em $29,3 \%$ dos pacientes. Observamos que a extensão do procedimento cirúrgico (biópsia versus ressecção parcial ou total) não proporciona diferença de sobrevida nos pacientes com AA e GBM. O que nossos resultados sugerem é que os resultados dependem da localização da lesão, da extensão do edema peritumoral e do estado clínico do paciente no pré-operatório.

Gamburg e col. ${ }^{8}$ avaliaram a influência do desvio da linha média na sobrevida de 114 pacientes com GBM que foram tratados com ressecção cirúrgica e radioterapia e concluíram que a presença de desvio da linha média pode ser considerada fator prognóstico independente. Em nosso estudo para estabelecer correlação entre a presença do desvio da linha média maior que $1 \mathrm{~cm}$ e sobrevivência entre pacientes tratados com AP observamos que esse desvio foi um fator de risco estatisticamente significativo para sobrevida mais curta, enquanto que a ausência de desvio de linha média após o tratamento com AP pode ser considerada prognóstico favorável.

Stummer ${ }^{24}$ concluiu que edema vasogênico é um fator prognóstico para o meningioma e metástases cerebrais, mas não para os gliomas. Outro estudo, ${ }^{9}$ com 48 pacientes com GBM, concluiu que o grau de edema peritumoral é um fator significativo na sobrevida global. Nosso estudo constatou que o edema peritumoral em tumores iniciais e recidivantes pode ser um importante fator prognóstico para resposta terapêutica ao AP. Observamos também que AA e GBM se assemelham com padrões idênticos na configuração do edema cerebral vasogênico. Dados de nossos resultados nos permitem considerar como uma resposta negativa ao tratamento com $\mathrm{AP}$ a regressão do volume tumoral sem a regressão do edema peritumoral. Consideramos como resposta positiva a regressão tumoral concomitante à redução do edema peritumoral independentemente do grau histológico do tumor.

Zhao e col. ${ }^{26}$ demonstraram que aumento da expressão da H-Ras e da atividade da via de sinalização Ras/ Raf/MEK contribui para o crescimento invasivo dos astrocitomas. Nosso estudo clínico com a administração intranasal do AP, um inibidor da Ras, para pacientes com gliomas recidivos, vem obtendo resultados de neuroimagem sugestivos de atividade antitumoral. No entanto, não consideramos como resposta positiva imagem de regressão tumoral sem a concomitante regressão do edema peritumoral. Poderá ser esperar demasiado que terapia anti-Ras aplicada de maneira isolada seja bem-sucedida contra os gliomas de alto grau. Deve-se levar em conta que esses tumores contêm inter-relação entre outras cascatas de sinalização ativadas além da Ras-RAF-Mek-ERK.

\section{Conclusão}

Os resultados obtidos em nosso estudo sugerem que: (1) localização tumoral, presença de edema e presença de desvio de linha média podem ser consideradas fatores preditivos de sobrevida em pacientes com gliomas malignos recidivos independentemente de seu grau histológico; (2) pacientes com tumores localizados nos gânglios basais apresentam sobrevida maior que aqueles com localização lobar; (3) pacientes com OA apresentam melhor resposta ao tratamento com AP intranasal que pacientes com glioblastoma ou AA.

Em conclusão, nosso estudo demonstrou que pacientes com gliomas profundos podem sobreviver significativamente mais do que aqueles com localização lobar. Além disso, a presença de edema peritumoral e o desvio da linha média foram correlacionados signifi- 
cativamente com menor tempo de sobrevida global no grupo de pacientes investigados. Isso pode corroborar a teoria de invasividade dos gliomas em diferentes microambientes do cérebro e pode ser um dado prognóstico de sobrevida. Um melhor entendimento da influência dos tumores no microambiente poderia conduzir a terapias que têm por alvo células geneticamente estáveis que circundam os gliomas, em vez de próprias células tumorais geneticamente instáveis, o que invariavelmente desenvolve resistência ao tratamento. A esse respeito, o monoterpeno AP tem atividade antitumoral por si só, mas em outra perspectiva vislumbramos sua utilização como solvente, permitindo a aplicação combinada de outros agentes quimioterápicos, por intermédio de uma única via de administração.

\section{Referências}

1. Arismendi-Morillo. Tumoral micro-blood vessels and vascular microenvironment in human astrocytic tumors. A transmission electron microscopy study. J Neurooncol. 2005;73:211-7.

2. Billottet C, Jouanneau J. Tumor-stroma interactions. Bull Cancer. 2008;95:51-6.

3. Cassoni P, Senetta R, Castellano I, Ortolan E, Bosco M, Magnani I, Ducati A. Caveolin-1 expression is variably displayed in astroglial-derived tumors and absent in oligodendrogliomas: concrete premises for a new reliable diagnostic marker in gliomas. Am J Surg Pathol. 2007;5:760-9.

4. Da Fonseca CO, Landeiro JA, Clark SS, Quirico-Santos T, Da Costa Carvalho MG, Gattass CR. Recent advances in the molecular genetics of malignant gliomas disclose targets for antitumor agent perillyl alcohol. Surg Neurol. 2006;65:52-8.

5. Da Fonseca CO, Masini M, Futuro D, Caetano R, Gattass RC, Quirico-Santos T. Anaplastic oligodendroglioma responding favorably to intranasal delivery of perillyl alcohol: a case report and literature review. Surg Neurol. 2006;66:611-5.

6. Da Fonseca CO, Schwartsmann G, Fischer J, Nagel D, Futuro D, Quirico-Santos T, Gattass CR. Preliminary results from a phase I/II study of perillyl alcohol intranasal administration in adults with recurrent malignant gliomas. Surg Neurol. 2008;70:259-66.

7. Da Fonseca CO, Linden R, Futuro D, Gattass CR, QuiricoSantos T. Ras pathway activation in gliomas: a strategic target for intranasal administration of perillyl alcohol. Arch Immunol Ther Exp (Warsz). 2008;56:267-76.

8. Gamburg ES, Regine WF, Patchell RA, Strottmann JM, Mohiuddin M, Young AB. The prognostic significance of midline shift at presentation on survival in patients with glioblastoma multiforme. Int J Radiat Oncol Biol Phys. 2000; 48:1359-62.

9. Hammoud MA, Sawaya R, Shi W, Thall PF, Leeds NE. Prognostic significance of preoperative MRI scans in glioblastoma multiforme. J Neurooncol. 1996;27:65-73.

10. Harpold HL, Alvord Jr. EC, Swanson KR. The evolution of mathematical modeling of glioma proliferation and invasion. J Neuropathol Exp Neurol. 2007;66:1-9.
11. Hoelzinger DB, Demuth T, Berens ME. Autocrine factors that sustain glioma invasion and paracrine biology in the brain microenvironment. J Natl Cancer Inst. 2007;99:1583-93.

12. Kostic A, Mihailovic D, Veselinovic S, Tasic G, Radulovic D, Stefanovic I. Peritumoral edema and karyometric variables in astrocytoma of the brain. J BUON. 2007;12:239-43.

13. Laws ER, Shaffrey ME, Morris A, Anderson Jr. FA. Surgical management of intracranial gliomas-does radical resection improve outcome? Acta Neurochir. 2003;85:47-53.

14. Lefranc F. Glioblastomas are resistant to apoptosis but less resistant to the autophagic process. Bull Mem Acad R Med Belg. 2007;162:331-8.

15. Mentrikoski M, Johnson MD, Korones DN, Scott GA. Glioblastoma multiforme in skin: a report of 2 cases and review of the literature. Am J Dermatopathol. 2008;30:381-4.

16. Mitchell P, Ellison DW, Mendelow AD. Surgery for malignant gliomas: mechanistic reasoning and slippery statistics. Lancet Neurol. 2005;4:413-22.

17. Nutt CL, Mani DR, Betensky RA, Tamoio P, Cairncross JG, Ladd C, et al. Gene expression-based classification of malignant gliomas correlates better with survival than histological classification. Cancer Res. 2003;63:1602-7.

18. Padma MV, Said S, Jacobs M, Hwang DR, Dunigan K, Satter $M$, et al. Prediction of pathology and survival by FDG PET in gliomas. J Neurooncol. 2003;3:227-37.

19. Pathy S, Jayalakshmi S, Chander S, Singh R, Julka PK, Rath GK. Prognostic factors influencing the outcome of thalamic glioma. Neurol India. 2002;50:37-40.

20. Pavlićević G, Roganović Z. Outcome of surgical treatment of malignant astrocytoma of the brain. Vojnosanit Pregl. 2003;60:147-54.

21. Ramnarayan R, Dodd S, Das K, Heidecke V, Rainov NG. Overall survival in patients with malignant glioma may be significantly longer with tumors located in deep grey matter. J Neurol Sci. 2007;15:49-56.

22. Stadlbauer A, Nimsky C, Gruber S, Moser E, Hammen T, Engelhorn $\mathrm{T}$, et al. Changes in fiber integrity, diffusivity, and metabolism of the pyramidal tract adjacent to gliomas: a quantitative diffusion tensor fiber tracking and MR spectroscopic imaging study. AJNR. 2007;28:462-9.

23. Swanson KR, Bridge C, Murray JD, Alvord Jr. EC. Virtual and real brain tumors: using mathematical modeling to quantify glioma growth and invasion. J Neurol Sci. 2003;15:1-10.

24. Stummer W. Mechanism of tumor-related brain edema. Neurosurg Focus. 2007;15:22(5):E8.

25. Wick W, Kuker W. Brain edema in neurooncology: radiological assessment and management. Onkologie. 2004;27:261-6.

26. Zhao Y, Xiao A, Dipierro CG, Abdel-Fattah R, Amos S, Redpath GT, et al. H-Ras increases urokinase expression and cell invasion in genetically modified human astrocytes through Ras/Raf/MEK signaling pathway. Glia. 2008; 56:917-24.

Original recebido em janeiro de 2009

Aceito para publicação em agosto de 2009

Endereço para correspondência

Clóvis Orlando da Fonseca

Unidade de Pesquisa Clínica

Serviço de Neurocirurgia, Departamento de Cirurgia Geral e Especializada.

Hospital Universitário Antônio Pedro

24030-210 - Niterói, $R J$

E-mail:clovis.orlando@uol.com.br; clovisorlando@huap.uff.br 